\title{
Minicloud: uma nuvem gratuita baseada em Power
}

\author{
Claudio d. Santos Júnior ${ }^{1}$, Lucas Wanner ${ }^{1}$, Sandro Rigo $^{1}$ \\ ${ }^{1}$ Instituto de Computação - Universidade Estadual de Campinas (UNICAMP) \\ Campinas - SP - Brasil \\ c195727@dac.unicamp.br,wanner@unicamp.br, srigo@unicamp.br
}

\begin{abstract}
Minicloud is a student-managed, free cloud service based on the IBM POWER architecture. The service is used by a variety of users worldwide for purposes ranging from building, porting, and testing Power systems and software and to conduct performance evaluation and academic studies. The Minicloud infrastructure is based on OpenStack and is managed and maintained by a team of undergrad students. This paper presents an overview of Minicloud, its configuration and available services, user profiles, and selected use cases.
\end{abstract}

Resumo. Minicloud é um serviço de nuvem gratuito gerenciado por alunos baseada na arquitetura de processadores POWER da IBM. O serviço é usado por uma variedade de usuários em todo o mundo para fins que vão desde a construção, porte e testes de sistemas e software para Power, até a avaliação de desempenho e estudos acadêmicos. A infraestrutura da Minicloud é baseada no OpenStack, gerenciada e mantida pelo nosso time de estudantes de graduação. Este artigo apresenta uma visão geral da Minicloud, sua configuração, serviços disponíveis, perfis de usuário e casos de uso.

\section{Introdução}

A Minicloud (https://openpower.ic.unicamp.br/minicloud/) é um serviço de nuvem gratuita disponível para usuários do mundo todo. O serviço é mantido e operado por alunos da graduação do Instituto de Computação da Unicamp, sendo um dos projetos do Laboratório OpenPower [5]. O laboratório é mantido através de um convênio entre a Unicamp e a IBM, sendo coordenado por professores da Universidade e funcionários da IBM.

O projeto foi criado em 2014, quando os usuários solicitavam, por e-mail, uma máquina virtual (MV) que era criada manualmente através do PowerKVM e as credenciais de acesso eram enviadas ao solicitante. Durante o período de criação do projeto, surgiram pelo mundo algo em torno de 10 projetos semelhantes ao da Minicloud. Apesar disso, atualmente só existem a Minicloud no Brasil e um outro projeto nos EUA. Em 2016, ganhamos o prêmio "Best in Show" no "Innov8 with POWER8 University Challenge" [1].

À medida que o serviço crescia, começou a ser necessário pensar em uma estratégia para automatizar o processo. Nesse momento surgiu a ideia de utilizar o OpenStack, que é capaz de gerir os múltiplos servidores e virtualizá-los em um único ambiente virtual. Além disso, ao criar uma conta nesse ambiente, o usuário possui uma quota para criar e destruir MV. Após colocarmos o OpenStack em produção, criamos diversos processos automatizados dentro da Minicloud. Por fim, criamos um formulário para facilitar e padronizar as solicitações e criações de conta.

Este artigo apresenta uma visão geral da Minicloud, incluindo sua descrição técnica, perfis e casos de uso, e perspectivas para o futuro do serviço. 


\section{Descrição Técnica}

O OpenStack requisita uma estrutura que possua pelo menos um controlador primário e um serviço de rede de nêutron (nós de rede) [3]. Para construir o OpenStack na versão Train, foi utilizado Ubuntu Server 18.04 LTS em todos os nós. Para o nó controlador e mais 4 nós as configurações de hardware foram: IBM Power8 @ $3.5 \mathrm{GHz}, 128 \mathrm{~GB}$ de memória RAM e 1,5 TB de disco rígido (HD). Além deles, existem mais dois nós com as seguintes configurações: IBM Power9 @ 3.8 GHz, 256 GB de RAM e 4 TB de HD.

\section{Máquinas Virtuais}

O OpenStack é uma solução para infraestruturas de nuvens públicas e privadas que consiste em uma combinação de softwares Open Source, apresentando robustez e confiabilidade. Ele é altamente configurável, permitindo a existência de soluções que se adaptem aos recursos de hardware disponíveis, com poucos ou muitos nós [4]. O OpenStack é responsável por gerir mais de 20 milhões de núcleos ao redor do mundo, sendo uma solução utilizada por diversas empresas e organizações como: Blizzard Entertainment, Walmart, Ministério do Interior da França, entre outros [2]. A versão Train conta com aprimoramentos na segurança e avanços em: inteligência artificial, aprendizado de máquina e no gerenciamento dos recursos.

Foram criadas 5 opções de configurações para as máquinas virtuais para oferecer maior flexibilidade ao usuário, chamadas de: minicloud.tiny, minicloud.small, minicloud.medium, minicloud.large e minicloud.max. Cada uma das configurações possuem, respectivamente, 1 GB de RAM e 20 GB de disco, 2 GB de RAM e 40 GB de disco, 3 GB de RAM e 60 GB de disco, 4 GB de RAM e 80 GB de disco e 10 GB de RAM e 80 GB de disco.

Para que possa ser possível utilizar a Minicloud, é necessário solicitar uma conta. A solicitação é feita através de um formulário disponível no site da Minicloud, e será analisada a respeito do perfil de uso. Caso aprovada, um e-mail é enviado com as credenciais para utilizar a conta por até 30 dias.

A Minicloud apresenta suporte para: Debian, Ubuntu, CentOS, OpenSUSE e Fedora, todos para a arquitetura ppc64le e alguns para ppc64 (BE). A máquina virtual estará disponível para ser utilizada alguns segundos após o lançamento, via ssh.

\section{Uso e Perfil de Uso}

A Minicloud foi criada com o princípio de ser um ambiente para testes e desenvolvimento de softwares para a arquitetura Power. Além de oferecer a oportunidade da comunidade Open Source de conhecer a arquitetura Power.

\subsection{Número de Requisições}

Desde o lançamento do formulário para as solicitações de acesso, recebemos um total de 1328 requisições, dos quais 809 foram aceitos, 427 foram recusados e para 92 requisições foram solicitadas mais informações. Se formos fazer uma média no decorrer de todo esse tempo, temos por mês a quantidade de 28,5 solicitações. Além disso, desde a criação da Minicloud, em 2014, estima-se que já foram realizados mais de 2000 solicitações, sendo mais da metade de usuários em outros países como: Índia, EUA, México e entre outros. 


\subsection{Perfil do usuário}

Buscamos estabelecer um perfil de nosso usuário através da análise das requisições. Por se tratar de muitas, elas foram agrupadas em 7 categorias, sendo: Interesse em Linux, Desenvolvedores para Power, Testar e conhecer Power (Testar Power), Portar softwares para Power, Suporte a pesquisa acadêmica (Suporte Acadêmico), Interessados em usar servidores (Uso de servidores) e Outros.

Com base nessas categorias, conseguimos comprovar que estamos conseguindo atingir o nosso objetivo de ser um ambiente para testes e desenvolvimento de softwares para a arquitetura Power. Visto que, as categorias de Testar e conhecer Power e Portar softwares para Power, representam um grupo de usuários que desejam contribuir para a comunidade Power, aumentando o acervo de pacotes disponíveis. Como pode ser visto na Figura 1, essas categorias juntas obtêm quase 50\% das solicitações.

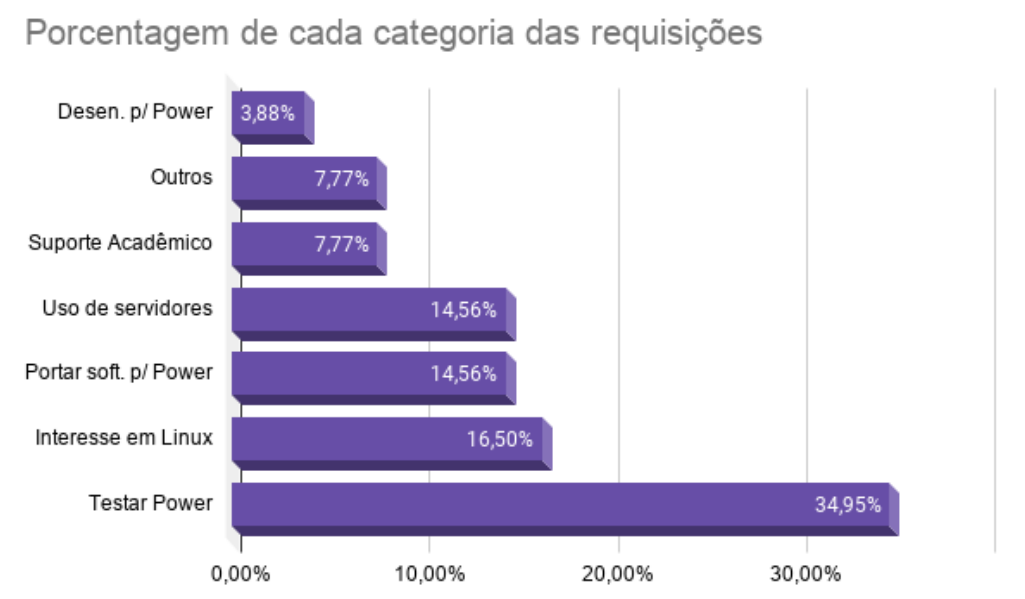

Figura 1. Percentagem de cada categoria das requisições

Já a categoria Interessados em usar servidores, representa usuários que desejam um ambiente remoto para realizar as suas atividades. No entanto, esse perfil não agrega a Minicloud, visto que existem provedores como o Google Cloud e a Microsoft Azure que podem fornecer servidores gratuitos, com um tempo de uso superior ao da Minicloud.

Além disso, a categoria Suporte a pesquisa acadêmica compõe o perfil dos usuários acadêmicos. O suporte a esses usuários pode ter sido dado de várias formas como: contas criadas para a realização de disciplinas sobre arquitetura de software, pesquisadores realizando testes de performance ou através do acesso a Minicloud ou ao servidor com GPU para a realização de algumas atividades que foram interrompidas ou paralisadas devido a pandemia do COVID-19.

\subsection{Solicitações Recusados}

Costuma-se recusar pedidos de acesso quando eles não envolvem ou não têm relação com a arquitetura Power. Por exemplo, a categoria Interesse em Linux costuma ser recusada, visto que há outras plataformas como Google Cloud, Virtual Box, terminal Linux para Windows 10, onde os usuários podem aprender sobre cloud e Linux. De forma semelhante, a categoria Interessados em usar servidores costuma ser recusada, pelos motivos já mencionados anteriormente. 


\section{Casos de uso e perspectivas}

No decorrer de todos esses anos, a Minicloud já gerou diversos resultados significativos para a comunidade. Esses resultados podem ser visualizados pelo grande número de projetos Open Source que utilizaram e utilizam a Minicloud. Alguns desses projetos são: Jenkins, gcc, Debian, Node JS, Eclipse, Julia, Alpine, entre outros.

Para contribuir ainda mais com a comunidade, é preciso que seja oferecido ainda mais recursos. Dessa forma, pretende-se realizar uma atualização na Minicloud, permitindo o uso de containers e GPU. A implementação dos containers será dada atualizandose a versão do OpenStack para um lançamento mais recente. Quanto ao acesso a GPU, o laboratório possui um servidor com essas características, o próximo passo é conseguir adicioná-lo como um nó ao OpenStack.

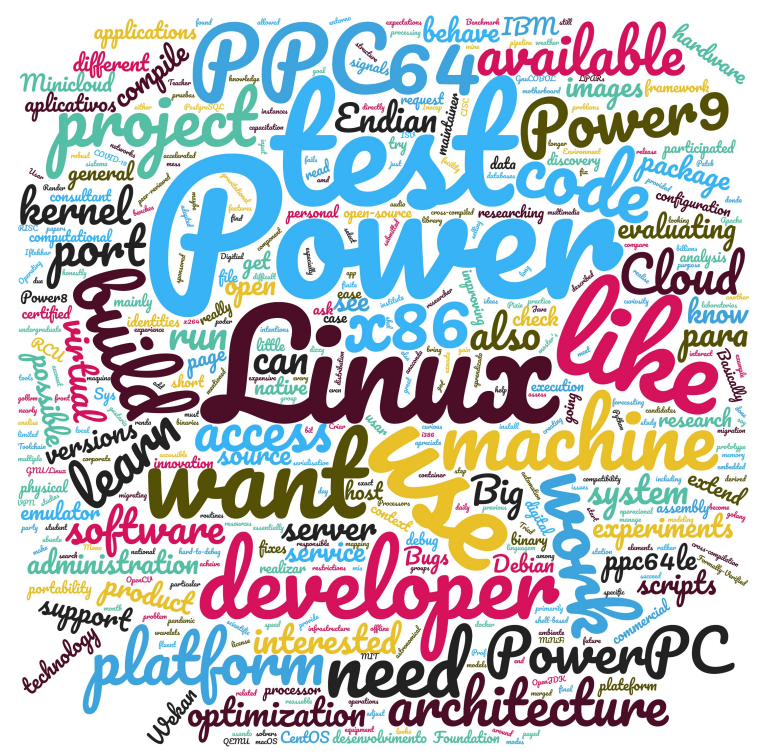

Figura 2. Principais palavras mencionadas nas solicitações

\section{Referências}

[1] OpenPOWER Fundation. With openpower, unicamp shares academic research across brazil. https://openpowerfoundation.org/with-openpower-unicamp-shares-academicresearch-across-brazil/.

[2] OpenStack. The most widely deployed open source cloud software in the world. https://www.openstack.org/.

[3] Anak Agung Putri Ratna, Tomi Wirianata, F. Astha Ekadiyanto, Ihsan Ibrahim, Diyanatul Husna, and Prima Dewi Purnamasari. Cloud computing network design for high performance computing implementation on openstack platform. In Proc. Intl. Conf. on Communication and Information Processing, ICCIP '17, page 356-360, 2017.

[4] Tiago Rosado and Jorge Bernardino. An overview of openstack architecture. In Proceedings of the 18th International Database Engineering \& Applications Symposium, IDEAS '14, page 366-367, 2014.

[5] Unicamp OpenPower Lab. Project website. https://openpower.ic.unicamp.br/. 